\title{
Características físicas, químicas e sensoriais de biscoitos elaborados com mistura de
}

\section{farinhas}

\author{
Physical, chemical and sensory characteristics of cookies made with a mixture of flours \\ Características físicas, químicas y sensoriales de las galletas elaboradas con una mezcla de harinas
}

Recebido: 18/04/2021 | Revisado: 25/04/2021 | Aceito: 27/04/2021 | Publicado: 10/05/2021

Izabela Fernanda Sirigatti Gouvea
ORCID: https://orcid.org/0000-0001-7864-2167
Instituto Federal de Educação, Ciência e Tecnologia do Sul de Minas Gerais, Brasil
E-mail: izabelafernanda1 @ outlook.com
Mariana Pereira Rezende Maciel
ORCID: https://orcid.org/0000-0003-1231-8895
E-mail: mariprmaciel@ @otmail.com
Elisângela Elena Nunes Carvalho
ORCID: https://orcid.org/0000-0002-1124-8066
Universidade Federal de Lavras, Brasil
E-mail:elisangelacarvalho@ dca.ufla.br
Marcelo Ângelo Cirillo
ORCID: https://orcid.org/0000-0003-2026-6802
Universidade Federal de Lavras, Brasil
E-mail: macufla@des.ufla.br
Brígida Monteiro Vilas Boas
ORducação, Ciência e Tecnologia do Sul de Minas Gerais, Brasi
ORCID: https://orcid.org/0000-0001-9010-2972
Onstituto Federal de Educação, Ciência e Tecnologia do Sul de Minas Gerais, Brasil
E-mail:brigida.monteiro@ifsuldeminas.edu.br
Aline Manke Nachtigall
ORCID: https://orcid.org/0000-0002-9691-0361
E-mail: aline.manke@ifsuldeminas.edu.br

\section{Resumo}

Os biscoitos caracterizam-se pela elevada aceitação e por permitirem o emprego de uma versatilidade de ingredientes em suas formulações. O objetivo deste estudo foi elaborar cookies com a mistura das farinhas de talos de beterraba (FTB), flocos de aveia (FA) e farinha de trigo (FT) e avaliar as características físicas, químicas e sensoriais dos mesmos. Os cookies foram desenvolvidos empregando-se o delineamento experimental centróide simplex, com um ponto central e restrição do limite máximo de farinha de trigo em 50\%. Nos biscoitos foi determinada a atividade de água (Aw), o pH, o $\mathrm{H}^{\circ}$, o $\mathrm{C}^{*}$ e a aceitação dos atributos sabor, textura e aspecto global. $\mathrm{O}$ modelo linear mostrou-se o mais adequado para explicar a variação da $\mathrm{Aw}$, do $\mathrm{pH}$ e de todos os atributos sensoriais, sendo que o quadrático conseguiu predizer as variações do $\mathrm{H}^{\circ}$ e do $\mathrm{C}^{*}$. A FTB apresentou efeito sinérgico sobre a Aw e antagônico sobre o pH, já o contrário foi observado para os FA. Os biscoitos elaborados com FTB apresentaram cor marrom e com menor pureza de cor, ao passo que os FA e a FT originaram biscoitos dourados e com maior pureza de cor. Os atributos sensoriais foram afetados negativamente pela presença da FTB. Portanto, a FTB apresentou influência marcante sobre os parâmetros físicos, químicos e sensoriais. No entanto, ressalta-se que quando a FTB foi empregada em até $17 \%$ da base farinácea obtiveram-se bons resultados.

Palavras-chave: Aproveitamento integral; Farinha de talo de beterraba; Flocos de aveia; Análise de cor; Aceitação.

\begin{abstract}
Cookies are characterized by high acceptance and allow the use of versatility of ingredients in their formulations. The aim of this study was to prepare cookies with the mixture of beet stalk flour (FTB), oat flakes (FA) and wheat flour (FT) and evaluate their physical, chemical and sensory characteristics. Cookies were developed using the simplex centroid experimental design, with a central point and restriction of the maximum limit of wheat flour by $50 \%$. In the cookies, water activity $(\mathrm{Aw}), \mathrm{pH}, \mathrm{H}^{\mathrm{o}}, \mathrm{C} *$ and acceptance of the attributes flavor, texture and overall appearance were determined. The linear model proved to be the most adequate to explain the variation in Aw, $\mathrm{pH}$ and all sensory attributes, the quadratic model was able to predict the variations in Ho and $\mathrm{C} *$. FTB had a synergistic effect on Aw and antagonistic on $\mathrm{pH}$, whereas the opposite was observed for FA. The cookies made with FTB were brown in color and with less color purity, while the FA and FT originated golden cookies and with greater color purity. Sensory attributes were negatively affected by the presence of FTB. Therefore, FTB had a marked influence on physical, chemical and
\end{abstract}


sensory parameters. However, it is noteworthy that when FTB was used in up to $17 \%$ of the meal base, good results were obtained.

Keywords: Full use; Beet stalk flour; oat flakes; Color analysis; Acceptance.

\section{Resumen}

Las galletas se caracterizan por una alta aceptación y permiten el uso de la versatilidad de ingredientes en sus formulaciones. El objetivo de este estudio fue preparar galletas con la mezcla de harina de remolacha (FTB), copos de avena (FA) y harina de trigo (FT) y evaluar sus características físicas, químicas y sensoriales. Las galletas se desarrollaron utilizando el diseño experimental centroide simplex, con un punto central y restricción del límite máximo de harina de trigo en un 50\%. En las galletas se determinó la actividad de agua (Aw), $\mathrm{pH}, \mathrm{H}^{\circ}, \mathrm{C} *$ y aceptación de los atributos sabor, textura y apariencia general. El modelo lineal resultó ser el más adecuado para explicar la variación en Aw, $\mathrm{pH}$ y todos los atributos sensoriales, el modelo cuadrático fue capaz de predecir las variaciones en $\mathrm{H}^{\circ}$ y $\mathrm{C}^{*}$. FTB tuvo un efecto sinérgico sobre Aw y un efecto antagonista sobre el pH, mientras que se observó lo contrario para FA. Las galletas elaboradas con FTB eran de color marrón y con menor pureza de color, mientras que las FA y FT originaron galletas doradas y con mayor pureza de color.Los atributos sensoriales se vieron afectados negativamente por la presencia de FTB. Por tanto, FTB tuvo una marcada influencia en los parámetros físicos, químicos y sensoriales. Sin embargo, cabe destacar que cuando se utilizó FTB hasta en un 17\% de la base de la comida, se obtuvieron buenos resultados.

Palabras clave: Uso completo; Harina de remolacha; Flocos de avena; Análisis de color, Aceptación.

\section{Introdução}

Biscoitos são produtos obtidos pelos processos de mistura, amassamento e cocção de farinhas diversas, podendo apresentar variabilidade nos recheios, textura, cobertura e formato (BRASIL, 2005). Dentre esses produtos, destacam-se os biscoitos tipo cookie, produtos de boa aceitação, consumidos por todas as faixas etárias, caracterizados como um bom veículo para incluir compostos bioativos. Apresentam excelente capacidade em abranger diversos ingredientes em sua formulação e são amplamente consumidos devido ao elevado Shelf Life e à textura nítida (Mareti, Grossmann \& Benassi, 2010).

A farinha do resíduo do processamento da beterraba ou de partes distintas das raízes tem sido muito estudadas, no sentido de reduzir o desperdício alimentar, e por considerar que quando há o consumo constante desta espécie, pode-se obter alguns benefícios a saúde, como auxilio na redução da pressão arterial, por ser um ótimo antioxidante natural, além de agir contra o envelhecimento precoce e, consequentemente, prevenir determinadas doenças relacionadas com o estresse oxidativo em humanos (Damodaran, Fennema \& Parkin, 2010; Bassetto, Samulak, Misugi, Barana \& Biancardi, 2011; Bassetto, Samulak, Misugi, Barana, \& Rosso, 2013; Costa, 2015; Crocetti et al., 2016).

Quanto à farinha de aveia, as $\beta$-glucanas, presentes na aveia apresentam atividade que favorece uma melhor modulação dos níveis de colesterol e glicemia, atuam retardando o esvaziamento gástrico e aumentando assim o tempo de contato dos nutrientes com a parede absortiva do intestino delgado, auxiliando na absorção dos nutrientes. Além disso, são de grande importância em panifícios, pois as $\beta$-glucanas melhoram a estabilidade e resistência da massa (Pisarro, Ronco \& Gotteland, 2014).

Ressalta-se ainda que, as farinhas mistas têm o objetivo de reduzir a dependência de importação de trigo, que se faz necessária devido a insuficiência da produção nacional. A substituição do trigo pelas farinhas mistas pode reduzir o custo do produto final, já que essas farinhas geralmente são desenvolvidas a partir do aproveitamento de outras matérias-primas, que além do custo reduzido, ainda apresentam o benefício de possuir maior valor nutricional (Cavallini, Carvalho, Brigide \& Maradini Filho, 2020).

Desta forma, o presente trabalho propôs avaliar o efeito da mistura das farinhas de talos de beterraba, flocos de aveia e farinha de trigo sobre as características físicas, químicas e sensoriais dos biscoitos. 


\section{Metodologia}

Para a realização deste estudo, utilizou-se uma metodologia de natureza quantitativa em modelo experimental. Pereira et al. (2018) caracterizam o método quantitativo, como aquele em que há coleta de dados quantitativos ou numéricos por meio do uso de medições de grandezas, as quais são analisados através de cálculos matemáticos.

Os biscoitos foram elaborados, na Cozinha Experimental do Instituto Federal de Educação, Ciência e Tecnologia do Sul de Minas Gerais (IFSULDEMINAS) - Campus Machado, com a mistura das farinhas de talo de beterraba (FTB - $X_{1}$ ), flocos de aveia $\left(\right.$ FA- $\left.X_{2}\right)$ e farinha de trigo $\left(\mathrm{FT}-X_{3}\right)$. Para tanto, empregou-se o delineamento experimental do tipo centróide simplex, no qual testou-se as misturas puras $\left(X_{1} ; X_{2} ; X_{3}\right)$, as binárias $\left(X_{1} . X_{2} ; X_{1} . X_{3} ; X_{2} X_{3}\right)$ e a ternária $\left(X_{1} X_{2} X_{3}\right)$, com igual proporção entre as farinhas (Tabela 1). O teor de farinha de trigo empregado foi limitado em no mínimo $50 \%$, ou seja, todas as formulações foram elaboradas com $50 \%$ ou mais desta farinha. Os componentes originais da mistura foram descritos em termos de pseudocomponentes e calculados conforme as equações a seguir: $x_{1}{ }^{\prime}=x_{1} / 0,50 ; x_{2}{ }^{\prime}=x_{2} / 0,50 ; x_{3}{ }^{\prime}=\left(x_{3}-0,50\right) / 0,50$. Para avaliar o erro experimental e testar os modelos, a mistura $X_{1} X_{2} X_{3}$ foi realizada em triplicata, portanto foram desenvolvidos 9 ensaios e 7 formulações.

Tabela 1. Planejamento centróide simplex para a formulação dos biscoitos.

\begin{tabular}{|c|c|c|c|c|c|c|c|}
\hline \multirow{3}{*}{ Ensaio } & \multirow{3}{*}{ Formulação } & \multicolumn{6}{|c|}{ Variáveis de Mistura } \\
\hline & & \multicolumn{3}{|c|}{ Componentes Originais } & \multicolumn{3}{|c|}{ Pseudocomponentes } \\
\hline & & FTB $\left(x_{1}\right)$ & FA $\left(\mathbf{x}_{2}\right)$ & FT $\left(\mathbf{x}_{3}\right)$ & FTB $\left(x_{1}^{\prime}\right)$ & FA $\left(x_{2}^{\prime}\right)$ & FT $\left(x_{3}{ }^{\prime}\right)$ \\
\hline 1 & 1 & 0,50 & 0,00 & 0,50 & 1 & 0 & 0 \\
\hline 2 & 2 & 0,00 & 0,50 & 0,50 & 0 & 1 & 0 \\
\hline 3 & 3 & 0,00 & 0,00 & 1,00 & 0 & 0 & 1 \\
\hline 4 & 4 & 0,25 & 0,25 & 0,50 & $1 / 2$ & $1 / 2$ & 0 \\
\hline 5 & 5 & 0,00 & 0,25 & 0,75 & 0 & $1 / 2$ & $1 / 2$ \\
\hline 6 & 6 & 0,25 & 0,00 & 0,75 & $1 / 2$ & 0 & $1 / 2$ \\
\hline 7 & $7(1)$ & 0,17 & 0,17 & 0,66 & $1 / 3$ & $1 / 3$ & $1 / 3$ \\
\hline 8 & $7(2)$ & 0,17 & 0,17 & 0,66 & $1 / 3$ & $1 / 3$ & $1 / 3$ \\
\hline 9 & $7(3)$ & 0,17 & 0,17 & 0,66 & $1 / 3$ & $1 / 3$ & $1 / 3$ \\
\hline
\end{tabular}

FTB - Farinha de talo de beterraba, FA- Flocos de aveia, FT - Farinha de trigo. Fonte: Autores (2021).

A farinha de talo de beterraba foi produzida a partir de talos de beterraba da cultivar Early Wonder (Beta vulgaris L.). Os flocos de aveia, a farinha de trigo e os demais ingredientes empregados na elaboração dos biscoitos foram adquiridos no comércio local de Machado/MG.

Os talos de beterraba, empregados na elaboração da farinha, foram selecionados, passaram pelo processo de limpeza em água corrente e seguiram para a sanitização em solução clorada (200 ppm/10 minutos). Os talos após o corte (transversalmente de $1 \mathrm{~cm}$ ) foram submetidos ao branqueamento (a vapor por 1 minuto) e passaram pelo processo de desidratação em secador de bandeja com circulação forçada de ar $\left(60^{\circ} \mathrm{C} / 6\right.$ horas). Os talos secos foram triturados em liquidificador industrial e a farinha tamisada em peneiras com abertura de malha de $250 \mu \mathrm{m}$. A farinha foi armazenada em sacos plásticos, ao abrigo da luz, umidade e calor até o momento do uso.

Os biscoitos foram produzidos seguindo os preceitos das boas práticas de fabricação, sendo as matérias primas pesadas individualmente, de acordo com as quantidades apresentadas na Tabela 2, e misturadas manualmente até a obtenção de 
uma massa homogênea. Após a moldagem (cilindro com $\varnothing$ de $2,5 \mathrm{~cm})$, a massa foi assada em forno industrial a gás $\left(180^{\circ} \mathrm{C} / 15\right.$ minutos), e resfriada em temperatura ambiente. Os biscoitos foram armazenados em potes de vidro hermeticamente fechados até o momento das análises.

Tabela 2. Formulação dos biscoitos tipo cookies.

\begin{tabular}{lc}
\hline Ingredientes & Quantidade (g) \\
\hline Farinha $^{\mathbf{1}}$ & 150 \\
Margarina com sal & 125 \\
Açúcar refinado & 125 \\
Ovos & 50 \\
Essência de Baunilha & 5 \\
Fermento químico & 2,5 \\
\hline
\end{tabular}

${ }^{1}$ As quantidades específicas das farinhas de talo de beterraba (FTB), flocos de aveia (FA) e farinha de trigo (FT) encontram-se na Tabela 1. Fonte: Autores (2021).

No Laboratório de Bromatologia do IFSULDEMINAS Campus Machado determinou-se a atividade de água por leitura direta em medidor de atividade de água Aqualab, da Decagon (AOAC, 2012), o pH com auxílio de pHmetro da Tecnal e a análise de cor por leitura, na superfície dos biscoitos, do ângulo hue e do Croma* com o colorímetro Minolta CR400, empregando o iluminante D65, com ângulo de observação de $2^{\circ}$ e no sistema de cor CIEL*a*b* (Minolta, 1998). Em cada análise utilizou-se cinco biscoitos por formulação.

No Laboratório de Análise Sensorial do IFSULDEMINAS Campus Machado, 54 consumidores não treinados, participaram do teste com consumidores para verificar a aceitabilidade dos atributos sabor, textura e aspecto global. Os consumidores, do sexo feminino e masculino, foram selecionados aleatoriamente por caracterizarem-se como consumidores de cookies e não possuíam alergia aos componentes da formulação dos biscoitos. A análise ocorreu após leitura e assinatura pelos participantes do Termo de Consentimento Livre e Esclarecido previamente aprovado junto ao projeto de pesquisa pelo Comitê de Ética da Universidade José do Rosário Avellano (CAAE 67445417.8.0000.5143). O teste foi conduzido em cabines individuais, onde as amostras (cerca de $5 \mathrm{~g}$ de biscoito) foram servidas, de forma balanceada, de três em três (em um único dia). Nas cabines os consumidores encontravam água a disposição e a ficha para a análise do produto, contendo uma escala hedônica estruturada de 9 pontos, ancorada nos extremos pelos termos "gostei extremamente" (9) e "desgostei extremamente" (1), de acordo Dustcosky (2013). O cálculo do índice de Aceitabilidade (IA) foi realizado de acordo com a equação IA (\%) = A x 100 / B, em que, A = nota média obtida para o produto e B = nota máxima dada ao produto (Teixeira, Meinert \& Barbetta, 1987).

Os modelos matemáticos para as variáveis resposta em função das variáveis de mistura foram examinados quanto à qualidade do ajuste, utilizando as estatísticas dadas pelo coeficiente de determinação $\mathrm{R}^{2}$, pela análise de variância do modelo e ajuste pelo teste F. A escolha dos modelos baseou-se no modelo matemático preditivo mais simples, com $\mathrm{R}^{2}>0,70$, com variância significativa para o modelo e não significativa para a falta de ajuste $(\mathrm{p} \leq 0,05)$. Para o estudo da significância dos efeitos individuais na variável resposta, as variáveis dependentes foram ajustadas em nível de 5\%, sendo as análises realizadas com o auxílio do programa Statistic 10,0. 


\section{Resultados e Discussão}

$\mathrm{Na}$ Tabela 3 são apresentados os valores de atividade de água, $\mathrm{pH}$ e análise de cor dos biscoitos elaborados com a mistura das farinhas.

A atividade de água dos biscoitos variou entre 0,29 e 0,55 (Tabela 3), o maior valor detectado foi na formulação com maior teor de farinha de talo de beterraba. Resultado similar foi obtido por Costa (2015), que verificou alta capacidade de retenção de água na farinha do resíduo de minimamente processados de beterraba. Já Lima, Câmara, de Oliveira, Alencar, Vasconcelos, Soares, Soares e Cavalcanti (2019), ao desenvolverem cookies elaborados com farinha de resíduo de frutas encontraram valores de atividade de água variando entre 0,43 e 0,49 .

Tabela 3. Valores médios para atividade de água, $\mathrm{pH}$ e análise de cor dos biscoitos

\begin{tabular}{|c|c|c|c|c|c|c|c|}
\hline \multirow{2}{*}{ Formulação } & \multicolumn{3}{|c|}{ Mistura $^{1}$} & \multirow{2}{*}{ Aw } & \multirow{2}{*}{ pH } & \multirow{2}{*}{ Hue } & \multirow{2}{*}{ Croma* } \\
\hline & FTB & FA & FT & & & & \\
\hline 1 & 0,50 & 0,00 & 0,50 & 0,54 & 5,97 & 42,03 & 10,74 \\
\hline 2 & 0,00 & 0,50 & 0,50 & 0,35 & 6,73 & 80,99 & 26,10 \\
\hline 3 & 0,00 & 0,00 & 1,00 & 0,28 & 6,45 & 80,27 & 33,22 \\
\hline 4 & 0,25 & 0,25 & 0,50 & 0,55 & 6,29 & 45,99 & 13,12 \\
\hline 5 & 0,00 & 0,25 & 0,75 & 0,29 & 6,64 & 79,28 & 30,36 \\
\hline 6 & 0,25 & 0,00 & 0,75 & 0,45 & 6,19 & 46,87 & 14,25 \\
\hline 7 & 0,17 & 0,17 & 0,66 & 0,40 & 6,26 & 49,80 & 17,58 \\
\hline
\end{tabular}

${ }^{1}$ Proporções reais de farinha de talo de beterraba (FTB), flocos de aveia (FA) e farinha de trigo (FT). Aw - atividade de água. Fonte: Autores (2020).

Quando se trata de biscoitos, a principal característica esperada é a baixa atividade de água, devendo essa ser inferior a 0,6 (Clerice, De Oliveira \& Nabeshima, 2013). Logo, os resultados obtidos mostraram-se satisfatórios, pois a atividade de água dos biscoitos não ultrapassou o valor de 0,6 , indicando que os biscoitos terão uma boa vida de prateleira, no que se refere a água disponível para as reações químicas, físicas e biológicas.

Assim como a atividade de água, o pH também está relacionado a conservação do alimento, envolvendo aspectos químicos, microbiológicos e enzimáticos. Os biscoitos desenvolvidos apresentaram valores de pH entre 5,97 e 6,73, estando os menores valores relacionados a presença da farinha de talo de beterraba nas formulações. A variação de $\mathrm{pH}$ observada por Lima et al. (2019) ao utilizarem farinhas de resíduos de frutas em biscoitos doces $(6,01$ a 6,36) está coerente com a verificada no presente estudo.

Os valores do ângulo Hue dos biscoitos variaram de 42,03a 80,99. Segundo o sistema CIELAB (Minolta, 1998), quando o ângulo Hue for $0^{\circ}$ corresponde a cor vermelha e $90^{\circ}$ equivale a cor amarela. Os biscoitos com maiores proporções de farinha de talo de beterraba apresentaram menor valor para o angulo Hue, ficando assim, mais próximos ao ângulo $0^{\circ}$, com a cor mais próxima de vermelho, enquanto as formulações com maiores percentuais de farinha de trigo e flocos de aveia situaram-se próximas ao ângulo $90^{\circ}$, ou seja, com cor amarela (Tabela 3).

Com relação à cromaticidade ou pureza da cor dos biscoitos, valores inferiores representam cores com menor saturação de pigmentos, enquanto valores superiores caracterizam-se em cores com maior saturação de pigmentos, ou mais puras. Os valores da pureza da cor dos biscoitos variaram de 10,74 a 33,22 (Tabela 3).

A presença das farinhas de trigo e flocos de aveia na formulação dos biscoitos deu origem a produtos com tonalidade amarelada e com maior saturação da cor, já a adição da farinha de talo de beterraba resultou em produtos com tonalidade 
amarronzada e com baixa saturação de cor (Tabela 3). Como não houve uma padronização na cor dos biscoitos, por exemplo com a possível adição de cacau na formulação, esse parâmetro pode ter interferido na aceitabilidade dos biscoitos.

Maciel, Gouvea, Cirillo, Vilas Boas e Nachtigall (2020), encontraram em biscoitos elaborados pela mistura das farinhas de talo de beterraba, flocos de aveia e farinha de trigo valores de L*, a* e b* variando entre 32,18 e 65,73; 4,08 a 12,04 e 7,20 a 32,73, respectivamente. Os autores verificaram que a farinha de talo de beterraba influenciou positivamente a cor vermelha dos biscoitos e negativamente a luminosidade, enquanto as outras duas farinhas contribuíram positivamente com a cor amarela e luminosidade dos cookies.

$\mathrm{Na}$ Tabela 4, são apresentados os coeficientes de regressão e os dados da análise dos modelos preditivos para a atividade de água, o pH e a análise de cor dos biscoitos. A mistura pura das farinhas influenciou significativamente todos os parâmetros físicos e químicos dos biscoitos, sendo que, a mistura binária da farinha de talo de beterraba com o farelo de aveia ou com a farinha de trigo contribuiu significativamente para a redução da tonalidade $\left(\mathrm{H}^{\circ}\right)$ e pureza da cor $(\mathrm{Croma})$. Observase ainda, que o coeficiente da mistura binária do farelo de aveia e da farinha de trigo $\left(\beta_{2} \beta_{3}\right)$ não apresentou efeito significativo sobre os parâmetros de cor analisados.

Ainda observando a Tabela 4, constata-se que as variações sobre os valores de atividade de água e sobre o $\mathrm{pH}$ dos biscoitos são explicadas pelo modelo preditivo linear $\left(\mathrm{R}^{2}-86,92 \%\right.$ e $\mathrm{R}^{2}-90,78 \%$, respectivamente). O modelo preditivo quadrático demonstrou ter bom ajuste e capacidade preditiva sobre os valores do ângulo Hue $\left(R^{2}-99,02 \%\right)$ e do Croma $\left(R^{2}\right.$ $99,84 \%)$.

Tabela 4. Coeficiente de Regressão e análise de variância do modelo ajustado à Aw, pH, valor L*, ângulo Hue e Croma.

\begin{tabular}{lcccc}
\hline \multirow{2}{*}{ Parâmetros } & \multicolumn{4}{c}{ Variáveis Respostas } \\
\cline { 2 - 5 } & $\mathbf{A w}$ & $\mathbf{p H}$ & ângulo Hue & Croma* \\
\hline$\beta_{1}$ & $0,5831^{*}$ & $5,9015^{*}$ & $42,5021^{*}$ & $10,7751^{*}$ \\
$\beta_{2}$ & $0,3656^{*}$ & $6,6868^{*}$ & $81,4604^{*}$ & $26,1350^{*}$ \\
$\beta_{3}$ & $0,2718^{*}$ & $6,4228^{*}$ & $80,7463^{*}$ & $33,2540^{*}$ \\
$\beta_{1} \beta_{2}$ & - & - & $-71,5837^{*}$ & $-21,8708^{*}$ \\
$\beta_{1} \beta_{3}$ & - & - & $-66,6273 *$ & $-31,5849^{*}$ \\
$\beta_{2} \beta_{3}$ & - & - & $-14,8804$ & 2,1264 \\
$\mathrm{R}^{2}$ & 0,8692 & 0,9078 & 0,9902 & 0,9984 \\
Modelo & $0,0022^{*}$ & $0,0008^{*}$ & $0,0033^{*}$ & $0,0002^{*}$ \\
Falta de ajuste & 0,0851 & 0,2767 & 0,0994 & 0,6673 \\
\hline
\end{tabular}

$\beta_{1}=$ porcentagem de farinha de talo de beterraba; $\beta_{2}=$ porcentagem de flocos de aveia; $\beta_{3}=$ porcentagem de farinha de trigo $(*=p<0,05)$; Aw - atividade de água. Fonte: Autores (2021).

Na sequência são apresentadas as equações que modelam numericamente o comportamento da mistura das farinhas de talo de beterraba $\left(X_{1}\right)$, flocos de aveia $\left(X_{2}\right)$ e farinha de trigo $\left(X_{3}\right)$ sobre os parâmetros físicos e químicos dos biscoitos.

$A w=0,5831 . F T B+0,3656 \cdot F A+0,2718 . F T$

$p H=5,9015 \cdot F T B+6,6868 \cdot F A+6,4228 \cdot F T$

$H^{o}=42,5021 . F T B+81,4604 \cdot F A+80,7463 . F T-71,5837 . F T B * F A-66,6273 . F T B * F T-14,8804 . F A * F T$

$C^{*}=10,7751 . F T B+26,1350 \cdot F A+33,2540 \cdot F T-21,8708 . F T B * F A-31,5849 . F T B * F T+2,1264 . F A * F T$ 
A região com as combinações entre as três variáveis $X_{1}, X_{2}$ e $X_{3}$ para a atividade de água, $\mathrm{pH}, \mathrm{H}^{\circ}$ e $\mathrm{C}^{*}$ pode ser observada nas curvas de contorno obtidas por meio dos modelos matemáticos ajustados e apresentadas na Figura 1.

Figura 1. Curvas de contorno para atividade de água (A), pH (B), ângulo Hue (C) e Croma* (D) dos biscoitos elaborados com a mistura das farinhas de talo de beterraba (FTB), flocos de aveia (FA) e farinha de trigo (FT).

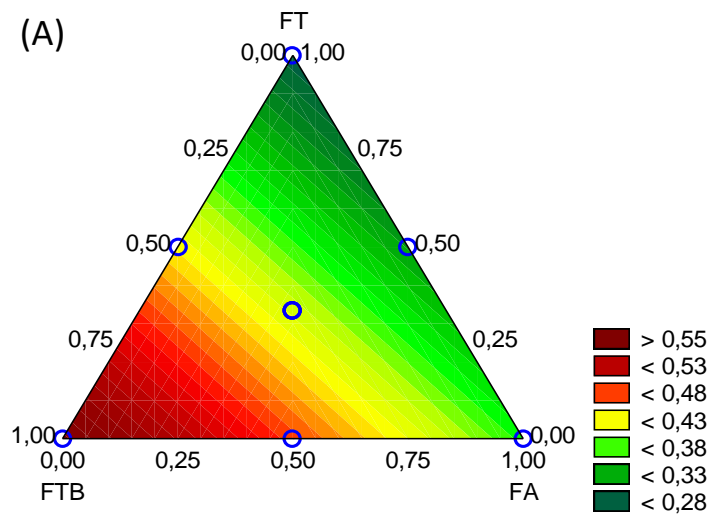

(C)

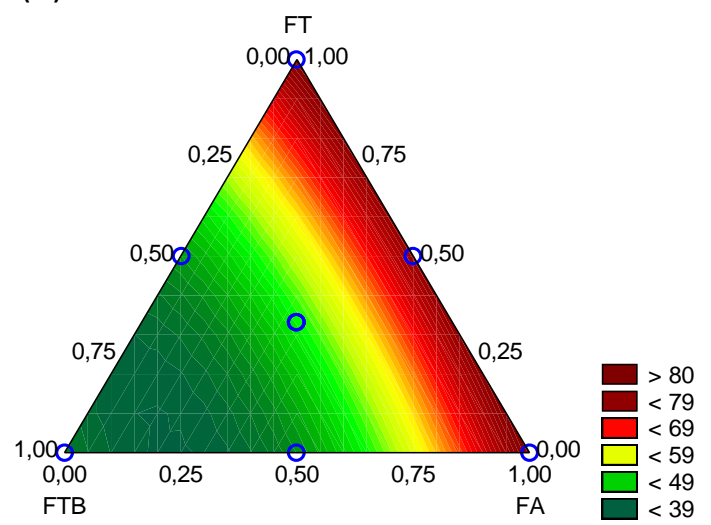

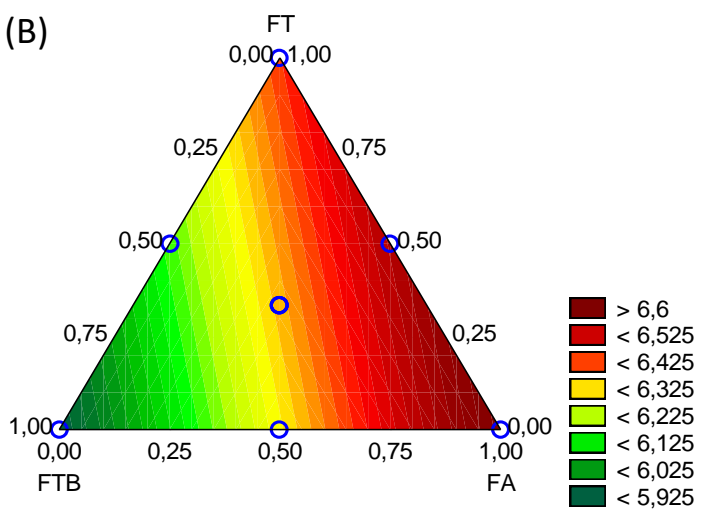

(D)

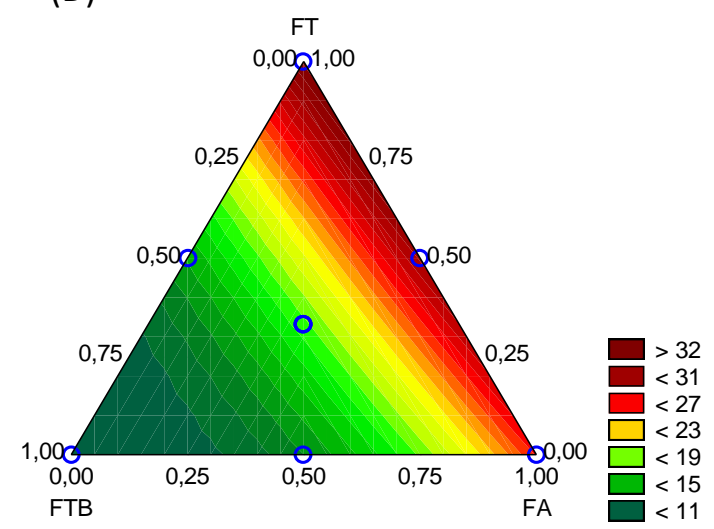

Fonte: Autores (2021).

Para a atividade de água, verifica-se um efeito sinérgico da farinha de talo de beterraba e antagônico da farinha de trigo, visto que a medida que se aproxima da região $100 \%$ farinha de talo de beterraba aumenta-se o valor da atividade de água dos biscoitos (região vermelha) e ao aproximar-se da região de $100 \%$ farinha de trigo reduz-se o valor do mesmo (região verde), como observado na Figura $1 \mathrm{~A}$.

A farinha de talo de beterraba apresentou um efeito antagônico sobre os valores de pH dos biscoitos, ou seja, quanto mais farinha de talo de beterraba contiver na formulação do biscoito menor será o valor do pH do mesmo. Ao contrário, a farinha de trigo e o farelo de aveia, apresentaram efeito sinérgico e contribuíram para o aumento do pH dos biscoitos (Figura 1B).

Ao observar as Figuras 1C e 1D, percebe-se claramente o mesmo comportamento nos parâmetros de cor avaliados $\left(\mathrm{H}^{\circ}\right.$ e C*). Na região próxima a $100 \%$ de farinha de talo de beterraba visualizam-se os menores valores para tonalidade e pureza da cor dos biscoitos, que aumentam ao aproximar-se das regiões de 100\% farelo de aveia e 100\% farinha de trigo.

Na Tabela 5 são apresentados os valores médios da aceitação dos atributos sabor, textura e aspecto global dos biscoitos elaborados pela mistura das farinhas. Os escores para a aceitação do atributo sabor variaram entre 4,54 e 7,46, a avaliação da textura oscilou entre 5,05 e 7,89, e o aspecto global de 5,00 a 7,50. Apenas o sabor do biscoito com maior teor de farinha de talo de beterraba (F1 - 50\% de FTB) foi classificado abaixo da zona de indiferença da escala, os demais atributos, 
independente da formulação, foram categorizados entre os termos hedônicos "nem gostei/nem desgostei" e "gostei moderadamente".

Tabela 5. Valores médios da aceitação para sabor, textura e aspecto global dos biscoitos.

\begin{tabular}{|c|c|c|c|c|c|c|}
\hline \multirow{2}{*}{ Formulação } & \multicolumn{3}{|c|}{ Mistura $^{1}$} & \multirow{2}{*}{ Sabor } & \multirow{2}{*}{ Textura } & \multirow{2}{*}{ Aspecto Global } \\
\hline & FTB & FA & FT & & & \\
\hline 1 & 0,50 & 0,00 & 0,50 & 4,54 & 5,05 & 5,00 \\
\hline 2 & 0,00 & 0,50 & 0,50 & 6,72 & 6,48 & 6,50 \\
\hline 3 & 0,00 & 0,00 & 1,00 & 7,30 & 7,65 & 7,50 \\
\hline 4 & 0,25 & 0,25 & 0,50 & 5,81 & 5,85 & 6,06 \\
\hline 5 & 0,00 & 0,25 & 0,75 & 7,46 & 7,89 & 7,46 \\
\hline 6 & 0,25 & 0,00 & 0,75 & 6,11 & 6,20 & 6,11 \\
\hline 7 & 0,17 & 0,17 & 0,66 & 6,98 & 7,12 & 7,02 \\
\hline
\end{tabular}

${ }^{1}$ Proporções reais de farinha de talo de beterraba (FTB), flocos de aveia (FA) e farinha de trigo (FT). Fonte: Autores (2021).

Analisando os resultados para aceitação sensorial (Tabela 5), nota-se que os biscoitos elaborados com farinha de talo de beterraba podem ter sofrido influência do sabor marcante da beterraba, afetando a aceitação do atributo. Percebe-se ainda, uma associação das menores notas aos biscoitos elaborados com a farinha de talo de beterraba, essa diferenciação na aceitação da textura pode ter sido influenciada pela maior umidade da farinha do resíduo da beterraba, que pode ter acarretado em biscoitos mais macios. Estudar a redução na diferença de coloração (Tabela 3) e avaliar a textura dos biscoitos (Tabela 5) é um direcionamento para futuras pesquisas.

Antigo, da Silva, Bergamasco e Madrona (2020) ao empregarem o corante microencapsulado de beterraba (betalaínas) em iogurtes observaram escores para o atributo sabor entre 6,23 e 6,58, concordando em parte com os resultados observados nos biscoitos elaborados com a farinha de talo de beterraba (F1, F4, F6 e F7). Oliveira, Macêdo, da Silva, Polari, de Oliveira e Azerêdo, também, observaram uma tendência de redução na aceitação do sabor conforme aumentava-se a quantidade de beterraba em iogurtes funcionais elaborados com farinha de beterraba. Maciel et al. (2020), encontraram escores para a aceitação do atributo cor variando de 6,07 a 7,37, cujos os menores valores estavam associados a biscoitos mais escuros. Estes resultados corroboram a afirmação de que a cor e o sabor característico da beterraba podem ter comprometido a aceitação dos biscoitos.

No entanto, Clímaco, Souza, Seccadio e Freitas (2020) verificaram aceitação satisfatória e sem distinção estatística entre as formulações de biscoitos elaborados somente com farinha de trigo e naqueles em que ocorreu a substituição de 10 e $20 \%$ da farinha de trigo por uma farinha mista de batata e beterraba. Oliveira, Cesconetto, Schveitzer e Foppa (2013) ao elaborarem biscoitos com $67 \%$ de farinha de raiz de beterraba associada com chocolate amargo, encontraram média de aceitação de 8,7 na escala hedônica de nove pontos, sendo para o ponto "gostei muitíssimo" uma aceitação de 73,3\%, quando avaliados por crianças de 8 a 10 anos de idade.

Travalini, Farias, Mayer, Demiate e Barana (2014) ao elaborarem biscoitos com farelo de trigo (FT), farinha de folha de cenoura (FFC) e folha de beterraba (FFB) obtiveram valores médios para aceitação do sabor variando de 5 a 6 , sendo que a nota 5 foi observada no biscoito produzido com $67 \%$ de FFB e 33\% de FT, sendo a nota 6 atribuída ao biscoito contendo a mistura das três farinhas. Estes valores são próximos, porém superiores ao do presente trabalho. 
Souza e Mattanna (2019) verificaram boa aceitação em bolos (9\% de substituição) e pães (17\% de substituição) elaborados com farinha de talos de beterraba. Para o bolo os valores obtidos para sabor, aroma, textura, aparência e aceitação global, foram: 8,54, 8,08, 8,52, 8,24 e 8,54, respectivamente. O pão apresentou valores médios de 8,52, 7,94, 8,68, 8,78 e 8,40, para sabor, aroma, textura, aparência e aceitação global, nesta ordem. Concordando com os dados obtido nos biscoitos elaborado com as três farinhas associadas (F7).

Na Tabela 6 são apresentados os índices de aceitabilidade para os atributos cor, sabor, textura e aspecto global dos biscoitos elaborados com a mistura das farinhas de talo de beterraba, farelo de aveia e farinha de trigo.

Tabela 6. Índice de aceitabilidade (\%) para os atributos sabor, textura e aspecto global dos biscoitos.

\begin{tabular}{|c|c|c|c|c|c|c|}
\hline \multirow[t]{2}{*}{ Formulação } & \multicolumn{3}{|c|}{ Mistura $^{1}$} & \multirow{2}{*}{ Sabor } & \multirow{2}{*}{ Textura } & \multirow{2}{*}{ Aspecto Global } \\
\hline & FTB & $\mathbf{F A}$ & FT & & & \\
\hline 1 & 0,50 & 0,00 & 0,50 & 50,44 & 56,11 & 55,56 \\
\hline 2 & 0,00 & 0,50 & 0,50 & 74,67 & 72,00 & 72,22 \\
\hline 3 & 0,00 & 0,00 & 1,00 & 81,11 & 85,00 & 83,33 \\
\hline 4 & 0,25 & 0,25 & 0,50 & 64,56 & 65,00 & 67,33 \\
\hline 5 & 0,00 & 0,25 & 0,75 & 82,89 & 87,67 & 82,89 \\
\hline 6 & 0,25 & 0,00 & 0,75 & 67,89 & 68,89 & 67,89 \\
\hline 7 & 0,17 & 0,17 & 0,66 & 77,56 & 79,11 & 78,00 \\
\hline
\end{tabular}

${ }^{1}$ Proporções reais de farinha de talo de beterraba (FTB), flocos de aveia (FA) e farinha de trigo (FT). Fonte: Autores (2021).

As formulações com 25\% (F4 e F6) e 50\% (F1) de farinha de talo de beterraba, não apresentaram índices satisfatórios para aceitação dos atributos avaliados nos biscoitos, uma vez que Monteiro (1984) recomenda um mínimo de $70 \%$ para colocar um produto no mercado. No entanto, esta farinha quando associada as demais na mistura ternária (F7), ou seja, no biscoito com $17 \%$ de farinha de talo de beterraba, apresentou um índice de aceitabilidade variando de 77,56 \% a 79,11\% (Tabela 6).

Bassetto et al. (2013), encontraram índice de aceitabilidade de $85 \%$ ao empregarem $20 \%$ de farinha de resíduo do processamento de beterraba na elaboração de biscoitos cookies, resultado esse semelhante ao observado na formulação que empregou as três farinhas juntas.

Mareti, Grosman e Benassi (2010), verificaram aceitabilidade adequada ao empregarem 70,7\% da mistura de farinha de soja desengordurada e farelo de aveia e 29,3\% de farinha de trigo, concordando com os resultados observados no cookie que continha a farinha de aveia e de farinha de trigo na mesma proporção (F2), cujo índice de aceitabilidade variou de $72,00 \%$ a $74,67 \%$ (Tabela 6).

Já Dos Santos et al. (2011), perceberam boa aceitação em relação ao sabor, textura, aroma e impressão global em biscoitos de buriti, com e sem aveia, sendo que os melhores resultados estavam associados aos biscoitos que continham aveia em sua formulação, obtendo índices de aceitabilidade acima de 80\%. Clímaco et al. (2020) desenvolveram biscoitos de farinha mista de beterraba e batata e constataram boa aceitação, acima de $70 \%$ para cor, sabor, odor, textura e aceitação global para as três formulações testadas (ausência, $10 \%$ e $20 \%$ de farinha mista de beterraba e batata).

Os resultados nos levam a concluir que é possível substituir parcialmente a farinha de trigo por farinha de talo de beterraba e flocos de aveia em cookies, mas deve-se atentar para as concentrações empregada de forma a não prejudicarem a aceitação do produto, além de demonstrarem a importância do emprego do delineamento de mistura, no qual através de 
modelagem matemática é possível predizer os resultados que serão encontrados com as diferentes proporções das componentes de mistura.

Neste sentido, o modelo linear foi o que se mostrou mais efetivo ao predizer o efeito da mistura das farinhas sob a aceitação sensorial dos biscoitos, como pode ser constatado ao observar os elevados valores de $\mathrm{R}^{2}$, a significância do modelo e a falta de ajuste não significativa (Tabela 7).

Todos os coeficientes de primeira ordem $\left(\beta_{1}, \beta_{2}\right.$ e $\left.\beta_{3}\right)$ foram significativos independente do parâmetro analisado, demonstrando o efeito das misturas puras sob a aceitação do sabor, da textura e do aspecto global dos biscoitos.

Tabela 7. Coeficientes de Regressão e análise de variância do modelo ajustado ao sabor, textura e aspecto global dos biscoitos.

\begin{tabular}{lccc}
\hline \multirow{2}{*}{ Parâmetros } & \multicolumn{2}{c}{ Variáveis Respostas } \\
\cline { 2 - 4 } & Sabor & Textura & Aspecto global \\
$\beta_{1}$ & $4,8248^{*}$ & $5,1554^{*}$ & $5,1980^{*}$ \\
$\beta_{2}$ & $7,1136^{*}$ & $6,9702^{*}$ & $6,9388^{*}$ \\
$\beta_{3}$ & $7,6912^{*}$ & $8,0447^{*}$ & $7,7608^{*}$ \\
$\mathrm{R}^{2}$ & 0,8340 & 0,7596 & 0,7992 \\
Modelo & $0,0046^{*}$ & $0,0139^{*}$ & $0,0091^{*}$ \\
Falta de ajuste & 0,2074 & 0,4572 & 0,2976 \\
\hline
\end{tabular}

$\beta_{1}=$ porcentagem de farinha de talo de beterraba; $\beta_{2}=$ porcentagem de flocos de aveia; $\beta_{3}=$ porcentagem de farinha de trigo $(*=\mathrm{p}<0,05)$. Fonte: Autores (2021).

Na sequência são apresentadas as curvas de contorno para a aceitação dos atributos avaliados nos biscoitos. 
Figura 2. Curvas de contorno para a aceitação dos atributos sabor (A), textura (B), aspecto global (C) dos biscoitos elaborados com a mistura das (FTB), flocos de aveia (FA) e farinha de trigo (FT).
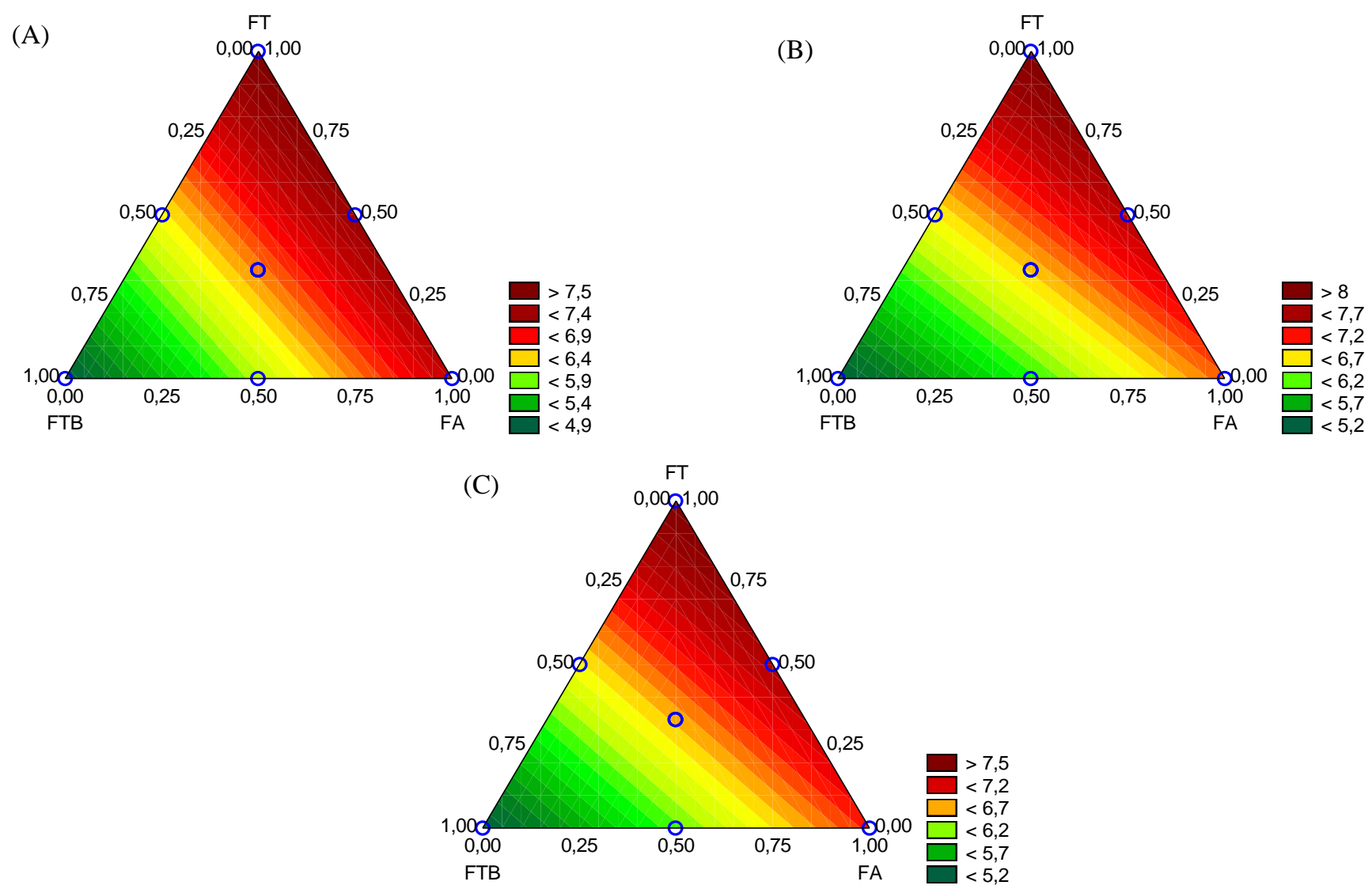

Fonte: Autores (2021).

Nas curvas de contorno, apresentadas na Figura 2, são apresentadas as combinações entre as três variáveis $X_{1}, X_{2}$ e $X_{3}$ para a aceitação dos atributos analisados nos biscoitos, de acordo com os modelos matemáticos ajustados apresentados na sequência.

Aceitação do sabor $=4,8248 \cdot F T B+7,1136 \cdot F A+7,6912 . F T$

Aceitação da textura $=5,1554 \cdot F T B+6,9702 . F A+8,0447 . F T$

Aceitação do aspecto global $=5,1980 . F T B+6,9388 . F A+7,7608 . F T$

Ao observar a Figura 2, comprova-se o efeito antagônico da farinha de talo de beterraba e um efeito sinérgico da farinha de trigo sobre a aceitação dos biscoitos, ou seja, a primeira esteve associada aos menores escores de aceitação (100\% de FTB na região verde), enquanto a segunda aos maiores escores (100\% de FT na região vermelha).

Tendo-se em vista que no experimento de misturas os resultados são gerados através de estatísticas pré-definidas pelo estudo base, é possível prever os resultados caso altere-se na formulação a concentração de um dos ingredientes. As vantagens deste delineamento são a redução do tempo de pesquisa e menores custos com testes, já que não se faz necessário reproduzir novos ensaios para obtenção de resultados, uma vez que se obtém um modelo de equação para o experimento. Portanto, a partir das equações dos modelos gerados neste estudo pode-se predizer o comportamento de qualquer variável resposta em função da modificação das proporções entre a farinha de talo de beterraba, os flocos de aveia e a farinha de trigo. 


\section{Conclusão}

A farinha de talo de beterraba teve uma influência marcante sobre os parâmetros físicos, químicos e sensoriais dos biscoitos. A mistura das farinhas de talo de beterraba, flocos de aveia e farinha de trigo mostrou-se viável na elaboração de biscoitos. Porém, a concentração da farinha de talo de beterraba deve ser limitada a no máximo $17 \%$ da base farinácea para não comprometer a aceitação do produto.

Sugere-se como continuidade desta pesquisa a elaboração de biscoitos empregando as mesmas farinhas, porém agregando cacau a formulação, visando a homogeneização da cor dos produtos e não permitir que esta diferença afete a aceitação global do produto.

\section{Agradecimentos}

Ao Instituto Federal de Educação, Ciência e Tecnologia do Sul de Minas Gerais - IFSULDEMINAS, pelo financiamento da pesquisa.

\section{Referências}

Antigo, J. L. D., Silva, J. M. da, Bergamasco, R. de C. \& Madrona, G. S. Microencapsulação de corante de beterraba (Beta vulgaris L.) utilizando maltodextrina e goma xantana como agentes encapsulantes e aplicação em iogurte. (2020). Research, Society and Development, 9 (12), e14091210896, 2020. 10.33448 / rsd-v9i12.10896. https://rsdjournal.org/index.php/rsd/article/view/10896.

Association of Analytical Chemists. (2012). Official methods of analysis of the association of official analytical chemists. 1(9a ed.), Gaithersburg.

Bassetto, R. Z., Samulak, R., Misugi, C., Barana, A. C. \& Rosso, N. (2013). Produção de biscoitos com resíduo do processamento de beterraba (Beta vulgaris L.). Revista Verde de Agroecologia e Desenvolvimento Sustentável, Mossoró, v. 8 (1), 139-145. https://dialnet.unirioja.es/servlet/articulo?codigo=7392975.

Bassetto, R. Z., Samulak, R., Misugi, C., Barana, A. C. \& Biancardi, C. R. (2011). Aproveitamento De Farinha De Resíduo De Beterraba Como Matéria prima Para Fabricação De Biscoito Tipo “Cookies”. Revista Technoeng, http://cescage.com.br/revistas/index.php/RTE/article/view/773/pdf.

BRASIL. Ministério da Saúde. Agência Nacional de Vigilância Sanitária. Resolução n 263, de 22 de setembro de 2005 . Regulamento técnico para produtos de cereais, amidos, farinhas e farelos. Diário Oficial da União, Poder Executivo, Brasília, 23 de setembro de 2005. Seção 1, p.368-369.

Cavallini, O. F., Carvalho, R. V., Brigide, P. \& Maradini Filho, A. M. (2020). Tópicos especiais em ciência e tecnologia de alimentos, v. 1, EDUFES. 334 p.: Farinhas Mistas utilizadas em produtos panificados: impotência tecnológica. cap 16. http://repositorio.ufes.br/bitstream/10/11618/1/digital_topicos-especiaisem-ciencia-e-tecnologia-de-alimentos.pdf\#page $=271$.

Clerice, M. T. P. S., De Oliveira, M. E. \& Nabeshima, E. H. (2013). Qualidade física, química e sensorial de biscoitos tipo cookies elaborados com a substituição parcial da farinha de trigo por farinha desengordurada de gergelim. Brazilian Journal of Food Technology, 16(2), 139-146. https://www.scielo.br/scielo.php?pid=S1981-67232013005000017\&script=sci_arttext\&tlng=pt.

Clímaco, C. N., Souza, M. L., Seccadio, L. L. \& Freitas, A. C. (2020). Análise fisico-quimica e sensorial de biscoito produzido com farinha mista de batata (Solanum Tuberosum L.) e beterraba (Beta Vulgaris L.). Research, Society and Development. 9(7). e943975204. https://rsdjournal.org/index.php/rsd/article/view/5204/4432.

Costa, A. P. D. (2015). Aproveitamento de resíduos de cenoura e beterraba da indústria de minimamente processados para elaboração de ingredientes funcionais. 2015. 97 f. Dissertação (Mestrado em Ciência e Tecnologia de Alimentos) - Universidade Federal do Rio Grande do Sul, https://www.lume.ufrgs.br/handle/10183/119753.

Crocetti, A., Ogleari, C. H., Gomes, G., Sare, I., Campos, F. R. \& Balbi, M. E. (2016). Determinação da composição centesimal a partir de s métodos de secagem para a produção da farinha de beterraba (Beta vulgaris, L. - família amaranthaceae). Visão Acadêmica, 17(4). https://revistas.ufpr.br/academica/article/view/51359.

Damodaran, S., Fennema, O. R. \& Parkin, K. L. (2010). Química de alimentos de Fennema. (4a ed.), Artmed.

Dos Santos, C. A., Ribeiro, R. C., Da Silva, E. V. C., Da Silva e Silva, N., Da Silva, B. A., Da Silva, G. F. \& De Barros, B. C. V. (2011). Elaboração de biscoito de farinha de buriti (Mauritia flexuosa L. f) com e sem adição de aveia (Avena sativa L.). Revista Brasileira de Tecnologia Agroindustrial, 5(1), 262273. https://periodicos.utfpr.edu.br/rbta/article/view/632.

Dutcosky, S. D. (2013). Análise sensorial de alimentos. (4a ed.), Champagnat. 531 p.

Lima, A. R. N., Câmara, G. B., de Oliveira, T. K. B, Alencar, W. D., Vasconcelos, S. H., Soares, T. da C., Soares, T. da C. \& Cavalcanti, M. da S. (2019). Caracterização físico-química e microbiológica de biscoitos confeccionados com farinha de resíduos de frutas. Research, Society and Development. 8(11), e198111452. https://rsdjournal.org/index.php/rsd/article/view/1452. 
Research, Society and Development, v. 10, n. 6, e39410515182, 2021

(CC BY 4.0) | ISSN 2525-3409 | DOI: http://dx.doi.org/10.33448/rsd-v10i5.15182

Maciel, M. P. R., Gouvea, I. F. S., Cirillo, M. A., Vilas Boas, B. M. \& Nachtigall, A. M. (2020). Estudo da mistura de farinhas de talos de beterraba, flocos de aveia e farinha de trigo e sua influência sob a cor de biscoitos tipo cookies. Brazilian Journal of Development, 6(4), 2062220636 .

Mareti, M. C., Grossmann, M. V. E. \& Benassi, M. De T. (2010). Características físicas e sensoriais de biscoitos com farinha de soja e farelo de aveia. Ciência e Tecnologia de Alimentos, 30(4), 878-883. https://www.scielo.br/scielo.php?pid=S0101-20612010000400007\&script=sci_abstract\&tlng=es.

Minolta. (1998). Precise color communication: color control from perception to instrumentation. Sakai.

Monteiro, C. L. B. (1984). Técnicas de avaliação sensorial. CEPPA.

Oliveira, L. P., Cesconetto, G., Schveitzer. B. \& Foppa, T. (2013). Avaliação e composição nutricional da farinha de beterraba e sua utilização no preparo de sobremesas. RIES, 2(1), 13-19. https://periodicos.uniarp.edu.br/index.php/ries/article/view/158.

Oliveira, J. M. de, Macêdo, L. T., Silva, H. M. F. das N. da; Polari, I. de L. B., Oliveira, F. L. N. de \& Azerêdo, G. A. Functional potential of beet yogurt. Research, Society and Development, 9(9), e196997153, 10.33448/rsd-v9i9.7153. https://rsdjournal.org/index.php/rsd/article/view/7153.

Pereira, A. S., Shitsuka, D. M., Parreira, F. J. \& Shitsuka, R. (2018). Metodologia da pesquisa científica. UFSM.

Souza, M. M. P. \& Mattanna, P. (2019). Desenvolvimento de produtos enriquecidos com farinha de talos de Beterraba. Revista Eletrônica Biociências, Biotecnologia e Saúde, n. 20, 16-26. https://interin.utp.br/index.php/GR1/article/view/2270.

STATSOFT. (2010). Statistica: data analysis software system. Version 10. Tulsa.

Teixeira, E., Meinert, E. M. \& Barbetta, P. A. (1987). Análise sensorial de alimentos. Editora UFSC.

Travalini, A. P., Farias, F. O., Mayer, R., Demiate, I. M. \& Barana A. C. (2014). Avaliação do efeito da incorporação de subprodutos agroindustriais na elaboração de Cookies. Revista Brasileira de Tecnologia Agroindustrial - UTFPR, Ponta Grossa, 8(2), 1592-1602. https://periodicos.utfpr.edu.br/rbta/article/view/1864. 